\title{
Individual Preferences on Grading Systems in Dental Schools
}

\author{
Mark Zmiyiwsky*, Nicholas Allen, Thomas Yoon, Kirk Zeller and Purushottam Lamichhane \\ School of Dental Medicine, Lake Erie College of Osteopathic Medicine, USA
}

*Corresponding author: Mark Zmiyiwsky, LECOM School of Dental Medicine, 4800

Received Date: September 17, 2018

Lakewood Ranch Blvd, Bradenton, FL 34211, USA.

\begin{abstract}
The objective of this study was to determine the type of grading system (pass/fail, pass/fail/pass with honors, traditional A-F grading) that individuals prefer in a sample of U.S. dental schools. Fifteen U.S. dental schools were selected, six of which used some type of pass/fail grading, the remainder used traditional grading and were randomly selected from a list of all U.S. dental schools. Six schools of the fifteen received the questionnaire which included demographic questions, questions on status as student or faculty, questions on current school grading system and individual preference for grading system types. Data was analyzed using an ANOVA with post hoc analysis using the Bonferroni method. Faculty significantly strongly disagree with pass/fail grading as compared to students ( $p=0.001$, CI 3.07-3.70). Additionally dental school faculty were more likely to disagree with the pass/fail/pass with honors grading system than first and second year dental students ( $p=0.001, \mathrm{CI} 2.74-3.38)$. Faculty agree significantly more with traditional grading compared to students. ( $\mathrm{p}=0.001, \mathrm{CI} 2.10-2.77$ ). Overall faculty prefer traditional grading and disagree with pass/fail grading of both types especially compared to first and second year students concerning pass/fail/pass with honors grading. This study will add to a growing body of research on the benefits, risks and effects of varying types of grading systems used in dental schools and their relation to the potential success of students both in dental education and those entering into dental postgraduate education programs.
\end{abstract}

Keywords: Dental education; Educational measurement; Education; Dental students; Dentistry; Schools; Dental

\section{Introduction}

In recent years there have been a variety of grading implements used in medical and dental professional education programs including traditional alphabetical and numerical systems, tiered ranking systems and simple pass/fail systems. Current research has found there to be little agreement in the type of grading system that should be used in academic healthcare institutions [1]. In addition there is relatively few data specifically regarding success or failure of differing grading system implementations in dental schools in the US.

As of August 2017, there are only eight U.S. dental schools that use some form of pass/fail grading criteria for student evaluation. Of those eight schools, four of them apply pass/fail criteria for grading while four of them use a pass/fail/pass with honors system [2]. According to the Commission on Dental Accreditation and the American Dental Association there are currently 777 fully accredited postgraduate advanced dental education programs in the United States and the latest data from the National Resident
Matching Program in 2017 shows that there were 5,070 medical residency match programs in the U.S $[3,4]$.

For candidates applying to medical residency programs, certain selection factors are evaluated by program directors in order to make an admissions decision on prospective residents. Recent research has shown that grades in clerkships, United States Medical Licensing Examination (USMLE) step one scores, grades in senior electives in specialty, number of honors grades and USMLE step two scores are the most important selection factors for medical residency programs [5]. Past research has found that in some cases directors for medical school residencies individually value a numeric grading system more than pass/fail systems, especially depending on which postgraduate program students are applying for [6-8]. However, data has highlighted the fact that pass/fail grading in medical school can help level the playing field for incoming professional students and prepare them to be effective, self-regulated learners [9]. In addition, pass/fail programs in medical school have been shown 
to create lower stress for students, increase student happiness, increase student satisfaction with education and home life as well as create more group cooperation among students $[1,8,10,11]$. Larger levels of stress, and emotional problems have been noted in medical schools using tiered grading systems of three or more levels compared to pass/fail grading systems as well $[11,12]$. Furthermore multiple studies have shown that despite changes in curriculum to pass/fail type grading systems there are little to no differences in student performance, residency placement and residency performance or national board examinations such as USMLE step one scores compared to students from a tiered or traditional grading evaluation system $[1,9,10,13,14]$.

Regarding postgraduate dental residency programs, similar factors are in place for program directors to use when selecting applicants to their respective programs. Across multiple dental residency programs interview ratings, clinical grades, dental school class rank, and National Board of Dental Examiners (NBDE) examination scores are considered majorly important selection factors [15-17]. Previously, NBDE examination scores were reported as numerical scores similar to USMLE scores, the grading reports for NBDE examination as of 2012, are reported only as pass or fail [18]. Surveys of program directors from multiple disciplines of dental residency programs note concern about changes in the NBDE examination score reporting citing that changes to pass/ fail grading for this national examination, that was once a strong selection criteria, will limit the ability for directors to evaluate candidates applying from pass/fail institutions that do not rank their own students $[16,17]$. Such pass/fail institutions that do not use GPA or class rank data force postdoctoral program directors to rely only on selection factors such as letters of recommendation, personal statements and interviews [19]. The reliance on nongraded measures of performance for students from pass/fail schools can strain students who may feel it necessary to go above and beyond to stand out as residency program candidates against other candidates from schools with traditional grading systems $[20,21]$. Additionally it has been noted that many dental residency program directors, with a lack of objective data on students from pass/fail schools, tend to abstain from reviewing applications from pass/fail institutions [20,21]. Despite the opinions dental residency program directors may share with medical residency program directors regarding the lack of objective data on students from pass/fail schools, there is limited evidence that there is no significant difference in achievement between dental students in pass/fail courses versus traditional grading courses [22]. Additionally educators from some dental residency programs that have admitted students from pass/fail dental programs have found that pass/fail students possess intelligence, high levels of professionalism, understanding, and empathy among other characteristics important to success in a specialty residency [23].

While a larger number of studies point toward the benefit of pass/fail grading systems in medical school education, there is still only a small portion of research data on the use of pass/fail grading in dentistry and more specifically how it affects students both in graduate dental education and postgraduate dental education. The objective of this study was to determine the type of grading system (pass/fail, pass/fail/pass with honors, traditional A-F grading) that individuals prefer in a sample of U.S. dental schools.

\section{Materials and Methods}

Data collection for this publication was designated exempt from the requirement for IRB approval per the exemption at 45 CFR 46.101(b)(2) for survey research of adults where the data are recorded in such a manner that human subjects cannot be identified. Approval was granted by the LECOM Institutional Review Board on August 3, 2017, Protocol 24-118.

Data was collected using an electronic questionnaire (SurveyMonkey.com, SurveyMonkey, San Mateo, CA, USA). Fifteen fully accredited U.S. dental schools were initially selected to receive the survey. The fifteen schools included the nine schools in the U.S. that used some type of pass/fail grading system in their educational curriculum, four of which use a pass/fail/pass with honors grading system and five of which use a pass/fail grading system. A comparable number of six schools using traditional grading were included and were randomly selected from a list of fifty-seven accredited U.S. dental schools, excluding those schools previously noted that use pass/fail grading systems [2,24]. Following selection of the fifteen schools, correspondence was made to the academic dean of each school. Of the fifteen schools selected, two pass/fail schools, two pass/fail/pass with honors schools and two traditional grading schools chose to participate in the survey following the initial correspondence and were sent an electronic questionnaire, to be distributed internally to all currently enrolled students and dental faculty at the respective institutions. The remaining nine schools of the total fifteen initially selected schools declined to participate in the study. Randomization of the selection of the non-pass/fail grading system schools was accomplished using the alphabetical list of fifty-seven U.S. dental schools in an electronic spreadsheet and randomized using =rand () function. A random number generator (RANDOM.ORG, Randomness and Integrity Services Ltd., Dublin, Ireland) was used to select six random numbers which were compared to the corresponding number of the school listed in the spreadsheet.

The questionnaire was programmed to bifurcate based on a required question determining the respondent's individual status as student or faculty (Figure 1). Certain questions in the survey were required to be answered in order to obtain appropriate sample size for data analysis while other demographic questions included in the questionnaire were optional. Respondents were asked a series of demographic questions including gender, age (for student), willingness to pursue postgraduate dental education (required question for student), experience with Pass/Fail grading in courses outside of dental school (for student), years of work in dental education (required for faculty) and whether a faculty member completed postgraduate dental education (required for faculty). Following the demographic questions, respondents were asked to rank, using a Likert scale, preference for a pass/fail grading system, a pass/fail/pass with honors system and a traditional (A-F) grading system in dental school. Data was collected without IP addresses in order to maintain anonymity of the participating schools in the electronic questionnaire and data was analyzed using an electronic spreadsheet. 


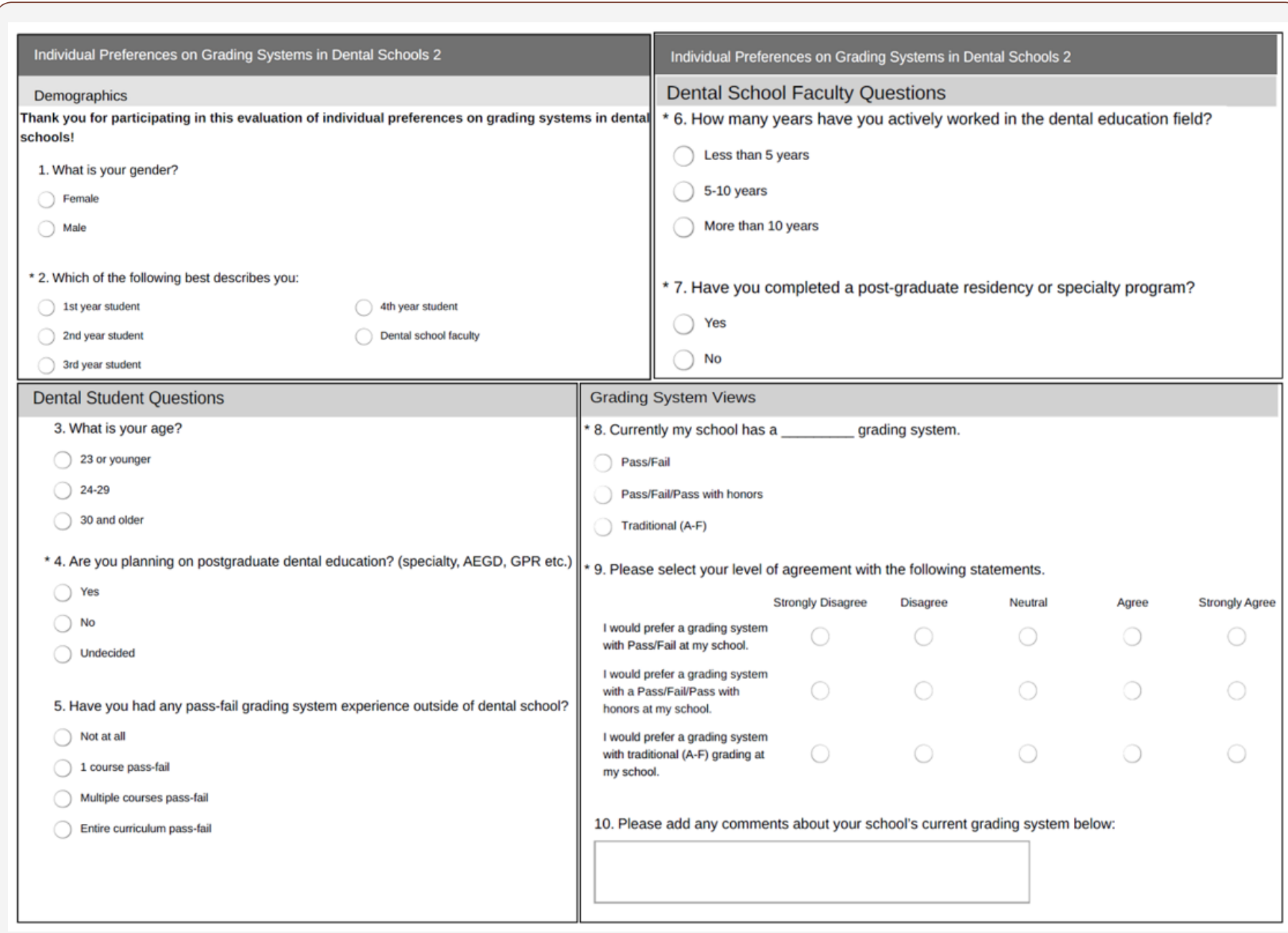

*Signifies question required to progress to end of survey.

Figure 1: Individual preferences on grading systems in dental schools students or faculty questionnaire.

Data was analyzed using an ANOVA with post hoc analysis using the Bonferroni method to compare individual preferences on grading systems against a range of demographic topics including: student age versus pass-fail coursework exposure, individual status versus preference for pass/fail grading, individual status versus preference for traditional grading, gender versus grading system preference, gender versus current grading system at respondent's institution, age versus grading system preference and pass/fail coursework exposure versus grading system preference.

\section{Results}

Of the six schools that were sent the questionnaire, total responses to individual questions were recorded. It should be noted again that questions two, four, six, seven, eight and nine were all required questions to be answered in order to complete the questionnaire.

Analysis of the questionnaire data revealed multiple significant relationships between demographic data collected and individual preferences for grading systems. An ANOVA with post hoc analysis using the Bonferroni method was used to determine if there was a significant relation between individual status versus preference for pass/fail grading, pass/fail/pass with honors grading and traditional grading.

A statistically significant relationship between individual status and pass/fail grading was found (Table 1), $F(4,341)=12.429$, p
$=0.001$. Post hoc analysis using the Bonferroni method further indicated that the mean score for dental school faculty $(M=3.39$, $\mathrm{SD}=1.365$ ) was significantly different than all of the four other status groups. More specifically it appears that dental school faculty disagreed with this grading method significantly more than dental students. When comparing individual status and preference for pass/fail/pass with honors grading a statistically significant relationship was found (Table 2), $F(4,345)=5.147, p=0.001$. Post hoc analysis using the Bonferroni method further indicated that the mean score for dental school faculty $(M=3.06, S D=1.426)$ was significantly different than first year $(M=2.38, S D=1.308)$ and second year students $(M=2.28, S D=1.241)$. It appears that dental school faculty disagreed with this grading method significantly more than first and second year students.

Table 1: Individual status versus preference for pass/fail grading system.

\begin{tabular}{|c|c|c|c|c|c|}
\hline Status & $\mathbf{N}$ & Mean & Std Dev & \multicolumn{2}{|c|}{ Confidence Interval } \\
\hline $1^{\text {st }}$ year student & 92 & 2.15 & 1.382 & 1.87 & 2.44 \\
\hline $2^{\text {nd }}$ year student & 88 & 2.08 & 1.157 & 1.83 & 2.32 \\
\hline $3^{\text {rd }}$ year student & 57 & 2.28 & 1.398 & 1.91 & 2.65 \\
\hline $4^{\text {th }}$ year student & 34 & 2.35 & 1.412 & 1.86 & 2.85 \\
\hline Dental School Faculty & 75 & 3.39 & 1.365 & 3.07 & 3.7 \\
\hline
\end{tabular}

${ }^{*} p=0.001$ 
Table 2: Individual status versus preference for pass/fail/pass with honors grading system.

\begin{tabular}{|c|c|c|c|c|c|}
\hline Status & $\mathbf{N}$ & Mean & Std Dev & \multicolumn{2}{|c|}{ Confidence Interval } \\
\hline $1^{\text {st }}$ year student & 92 & 2.38 & 1.308 & 2.11 & 2.65 \\
\hline $2^{\text {nd }}$ year student & 88 & 2.28 & 1.241 & 2.02 & 2.55 \\
\hline $3^{\text {rd }}$ year student & 57 & 2.82 & 1.212 & 2.5 & 3.15 \\
\hline $4^{\text {th }}$ year student & 34 & 2.41 & 1.131 & 2.02 & 2.81 \\
\hline $\begin{array}{c}\text { Dental School } \\
\text { Faculty }\end{array}$ & 79 & 3.06 & 1.426 & 2.74 & 3.38 \\
${ }^{*} \mathrm{p}=0.001$
\end{tabular}

Table 3: Individual status versus preference for traditional grading system.

\begin{tabular}{|c|c|c|c|c|c|}
\hline & $\mathbf{N}$ & Mean & Std Dev & \multicolumn{2}{|c|}{ Confidence Interval } \\
\hline $1^{\text {st }}$ year student & 92 & 3.48 & 1.33 & 3.2 & 3.75 \\
\hline $2^{\text {nd }}$ year student & 89 & 3.58 & 1.214 & 3.33 & 3.84 \\
\hline $3^{\text {rd }}$ year student & 56 & 3.52 & 1.452 & 3.13 & 3.91 \\
\hline $4^{\text {th }}$ year student & 34 & 3.26 & 1.399 & 2.78 & 3.75 \\
\hline $\begin{array}{c}\text { Dental School } \\
\text { Faculty }\end{array}$ & 78 & 2.44 & 1.473 & 2.1 & 2.77 \\
\hline
\end{tabular}

${ }^{*} p=0.001$

The relationship between individual status and preference for traditional grading was also found to be statistically significant (Table 3), $F(4,344)=9.489, p=0.001$. Post hoc analysis using the Bonferroni method further indicated that the mean score for dental school faculty $(M=2.44, S D=1.473)$ was significantly different than all other status groups. Specifically faculty were found to agree significantly more with traditional grading than all of the student groups.

\section{Discussion}

The literature focused on dental education contains relatively few data on the use of pass/fail grading in dentistry, the preferences of faculty and students in regards to pass/fail grading systems and how pass/fail grading systems affect students in graduate and postgraduate dental education in terms of whether such grading systems are successful in creating well-rounded students who are as competitive as those who have learned in institutions with traditional lettered or numerical grading systems.

Our study has demonstrated that dental school faculty significantly strongly disagree with pass/fail grading overall as a grading system compared to dental students. This may be due in part to the majority of dental school faculty likely having attending dental schools or worked in educational environments that participated in traditional grading as there are currently only nine dental schools in the U.S. out of sixty-six total that use a form of pass/fail grading in their curriculum [2,24]. Additionally the literature shows that program directors for some medical school residencies individually value a numeric grading system over pass/ fail systems and dental residency program directors are generally biased against dental students from pass/fail dental schools due to lack of objective measures of student performance [6,8,20-21].

Our study further demonstrated that dental school faculty strongly agree more with traditional grading compared to dental students. This could suggest that some dental students prefer to have some of the benefits that pass/fail grading, as opposed to traditional grading, has been shown to provide in studies of medical students such as a level playing field for incoming students, [9] lower stress levels and better group cooperation among peers, although more research on the psychological and social benefits of pass/fail grading in dental education need to be studied to confirm this [1,8,10-11,21]. Students that disagree with traditional grading, and therefore may prefer to be educated at a pass/fail type school, may be less competitive when applying to postgraduate dental education programs as these students may theoretically be seen as inferior in the eyes of postgraduate dental education programs directors [20-21]. However research has shown little difference in medical residency placement or medical resident performance in alumni from pass/fail programs as well as little difference in achievement among dental students in pass/fail dental programs compared to their traditionally graded counterparts $[1,9,13,22]$.

Additionally the study demonstrated that dental school faculty significantly disagree more with the pass/fail/pass with honors grading system compared to dental students, specifically in their first and second year. At present there are currently four U.S. dental schools applying the three-tiered grading system method, $\urcorner$ and available information suggests that this number and the total number of pass/fail dental schools has remained stable over time $[2,21]$. Still it should be noted that research in the medical education field has demonstrated that students in schools with three-tiered or higher grading systems had elevated levels of stress, rates of student burnout and emotional exhaustion compared to those schools using a pass/fail two-tiered grading system [12]. Further research on the psychological, social and academic benefits of a three-tiered grading system in dental education, especially in terms of postgraduate dental education program director acceptance of residents from a three-tiered grading system school as compared to traditional grading and pass/fail grading are needed to supplement currently available medical education research on the topic in order to better understand the role of three-tiered grading systems in the future of dental education.

Limitations of this study include the total sample size of schools that received the questionnaire. Sample size may have been significantly increased by sending the questionnaire to more dental programs. It would be interesting to repeat the study with a more strict data collection protocol and distribute the questionnaire to every dental school in the U.S. and possibly additional international dental education programs. Furthermore it would be interesting to conduct a survey study focusing specifically on postgraduate dental education program directors to determine their individual preferences regarding students applying to their programs from schools with pass/fail or pass/fail/pass with honors grading systems.

From the most recently available data on the 2016-2017 class of applicants to dental school, there was a 157 students enrollment increase above the previous year. This information coupled with the fact that there has been an ever increasing trend in the capacity to train new dentists over the last fifteen years shows that more and more individuals are choosing to pursue a career in dentistry [25]. As more and more students enter the world of dental education 
a choice between schools with different grading systems must be made. As time goes on, increases in research regarding the practical benefits and risks of multiple types of grading systems implemented in dental schools across the U.S. will give students better insight into the ideal dental education that will prepare them to successfully graduate, enter into postgraduate education programs if they so choose and ultimately become a successful practitioner.

\section{Conclusion}

This study identified individual preferences from dental students and dental school faculty regarding different grading systems used in U.S. dental schools. We found that dental school faculty strongly disagree with pass/fail grading as compared to students. We found that dental school faculty tend to significantly agree more with traditional grading compared to dental students. Additionally we found that dental school faculty tend to disagree more with a pass/fail/pass with honors grading system as compared to dental students, specifically those in their first and second year.

\section{Acknowledgement}

The authors would like to thank Dr. Katie Dinh for her advice on the project and Ms. Nikole Niemeyer for her work on and help with the statistical analyses.

\section{References}

1. Bloodgood RA, Short JG, Jackson JM, Martindale JR (2009) A change to pass/fail grading in the first two years at one medical school results in improved psychological well-being. Acad Med 84(5): 655-662.

2. (2015) Grading Resource Guide.

3. (2018) Search Engine: Search for Dental Programs. Commission on Dental Accreditation.

4. (2017) National Resident Matching Program. Results and Data: 2017 Main Residency Match.

5. Green M, Jones P, Thomas JX (2009) Selection criteria for residency: results of a national program directors survey. Acad Med 84(3): 362367.

6. Provan JL, Cuttress L (1995) Preferences of program directors for evaluation of candidates for postgraduate training. CMAJ 153(7): 919923.

7. Tardiff K (1980) The effect of pass-fail on the selection and performance of residents. J Med Educ 55(8): 656-661.

8. Spring L, Robillard D, Gehlbach L, Simas TA (2011) Impact of pass/fail grading on medical students' well-being and academic outcomes. Med Educ 45(9): 867-877.

9. White CB, Fantone JC (2010) Pass-fail grading: laying the foundation for self-regulated learning. Adv Health Sci Educ Theory Pract 15(4): 469477.
10. Rohe DE, Barrier PA, Clark MM, Cook DA, Vickers KS, et al. (2006) The benefits of pass-fail grading on stress, mood, and group cohesion in medical students. Mayo Clin Proc 81(11): 1443-1448.

11. Slavin SJ, Schindler DL, Chibnall JT (2014) Medical student mental health 3.0: improving student wellness through curricular changes. Acad Med 89(4): 573-577.

12. Reed DA, Shanafelt TD, Satele DW, Power DV, Eacker A, et al. (2011) Relationship of pass/fail grading and curriculum structure with wellbeing among preclinical medical students: a multi-institutional study. Acad Med 86(11): 1367-1373.

13. Vosti KL, Jacobs CD (1999) Outcome measurement in postgraduate year one of graduates from a medical school with a pass/fail grading system. Acad Med 74(5): 547-549.

14. McDuff SGR, Mcduff D, Farace JA, Kelly CJ, Savoia MC, et al. (2014) Evaluating a grading change at UCSD school of medicine: pass/fail grading is associated with decreased performance on preclinical exams but unchanged performance on USMLE step 1 scores. BMC Med Educ 14: 127.

15. Bell LT, Sukotjo C, Yuan JC, Johnson BR (2014) Applicant selection procedures in endodontic specialty programs in the United States: program director's perspective. J Endod 40(6): 797-804.

16. Majewski RF, da Fonseca MA, DeVries ES Hu JC, Murdoch-Kinch CA (2008) Factors influencing pediatric dental program directors' selection of residents and demographics of current directors. J Dent Ed 73(3): 338-344.

17. Khan S, Carmosino AJ, Chia-Chun Yuan J, Lucchiari N Jr, Kawar N, et al. (2015) Postdoctoral periodontal program directors' perspectives of resident selection. J Periodontol 86(2): 177-184.

18. Joint Commission on Dental Examinations (2011) Update on National Board Dental Examinations. American Dental Association, Chicago, US.

19. Brodie AJ, Crow HC, Eber RM, Handysides R, Holexa R, et al. (2011) Evaluating postdoctoral dental candidates: assessing the need and recommendations for a national qualifying examination. J Dent Educ 75(6): 719-725.

20. (2013) Advanced Dental Education Admissions. Evaluating applicants from pass-fail schools. Presented at: ADEA Councils of Students, Residents, and Fellows Interim Meeting, Oklahoma, US.

21. (2018) American Dental Education Association. Making the grade in a pass/fail environment: what it means for students.

22. Hall DL, Taft TB (1976) Pass/fail versus a-f grading: a comparative study. J Dent Educ 40(5): 301-303.

23. (2014) American Academy of Periodontology. Selecting our residents in the pass/fail environment. Presented at: American Academy of Periodontology Annual Meeting San Francisco, USA.

24. (2018) U.S. and Canadian Dental Schools.

25. Wanchek T, Cook BJ, Valachovic RW (2017) U.S. dental school applicants and enrollees, 2016 entering class. Journal of Dental Education 81(11): $1373-1382$. 